\title{
ORIGEN E HISTORIA DEL PROGRAMA COOPERATIVO CENTROAMERICANO Y DEL CARIBE PARA EL MEJORAMIENTO DE CULTIVOS ALIMENTICIOS Y PRODUCCIÓN ANIMAL
}

\author{
Alejandro Fuentes ${ }^{1}$, Carlos Salas $^{2}$, Angel Salazar $^{3}$
}

\section{PRIMERA REUNION DEL PCCMM TURRIALBA, COSTA RICA, 1954}

En 1941, por invitación de la Secretaría de Agricultura y Ganadería de México -SAG-, la Fundación Rockefeller envió una "Comisión de Estudio" para establecer las condiciones agrícolas en la República y para discutir con miembros de la Secretaría, las posibilidades de establecer un Programa Cooperativo para el mejoramiento de la producción de alimentos. Como resultado de estas deliberaciones se creó "La Oficina de Estudios Especiales, (OEE) y la Fundación Rockefeller" como una entidad de colaboración internacional en mejoramiento agrícola, la cual tuvo un éxito sorprendente con implicaciones importantes para otros países.

Dentro de esas implicaciones surgieron solicitudes a la Fundación Rockefeller para establecer programas de cooperación internacional en los países de Centroamérica y Panamá, así se estableció la necesidad de crear una organización técnica para mejorar la agricultura de esta región, habiendo a Fundación Rockefeller, encomendado esa misión al Dr. Edwin de J. WeIlhausen, en ese entonces, Director del Programa de Maíz de dicha oficina. El Dr. WeIlhausen con una clara visión de lo que se le encomendó, inmediatamente se puso en contacto con el personal técnico agrícola y Ministerios de Agricultura de Centroamérica y Panamá, logrando la creación de un pequeño Proyecto Centroamericano de Mejoramiento de Maíz "PCCMM", el cual realizó su primera reunión del 24 al 30 de octubre de 1954 en el Instituto Interamericano de Ciencias Agrícolas -IICATurrialba, Costa Rica. Este programa fue el punto de partida de lo que hoy es el Programa Cooperativo Centroamericano para el Mejoramiento de Cultivos Alimenticios y Producción Animal (PCCMCA).

En esta primera reunión se contó con la presencia de 61 representantes de Centroamérica y Panamá, así como con miembros de la Fundación Rockefeller, de IICA y con la presencia de observadores de México, Colombia y Venezuela, habiéndose presentado en esta reunión 78 trabajos, todos relacionados con el cultivo del maíz. Los organizadores de esta primera reunión, programaron entre otras actividades, la presentación por país, sobre datos ecológicos, económicos y una exposición de lo que se había realizado en mejoramiento. de maíz. Esta información, además, fue libremente intercambiada, sentando así las bases de un Programa Cooperativo, voluntario, planeado para ser dirigido y realizado por los mismos técnicos de la región, como lo es el PCCMCA en estos momentos.

Como hoy sabemos, este programa internacional es el único en Latinoamérica que ha tenido continuidad en sus 35 años de existencia, con el mismo número de reuniones anuales celebradas hasta 1989, por lo que creemos conveniente remontamos y tomar el pensamiento expresado por algunos personajes que asistieron a la inauguración de la primera reunión en 1954.

El Dr. Ralph H. Alle a la sazón Director del Instituto Interamericano de Ciencias Agrícolas, al referirse al maíz dijo: "Puedo ver en el futuro la posibilidad de un programa Regional, sobre el Mejoramiento del Maíz que ha sido establecido por ustedes en esta reunión..." Estas expresiones las exteriorizó el Dr. Alle con mentalidad pragmática, pues todos sabemos ahora lo versátil que es el maíz bajo el punto de vista social, cultural y utilitario, a la vez, con una mentalidad futurista predijo lo que hoy estamos haciendo, en forma ampliada a los principales cultivos alimentarios y agropecuarios. Gracias Dr. Alle por el aliento recibido en buena hora.

En esta primera reunión, el presidente Figueres, de Costa Rica, en su discurso de inauguración, hizo un análisis de los aspectos económicos, sociales y culturales de nuestros países y muy especialmente de Costa Rica, diciendo además, respecto a la identificación de nuestros pueblos con el maíz, debemos agregar y reconocer que la ciencia apunta que en un lugar de Mesoamérica tuvo su origen este preciado grano, que también ha tenido que ver con el desarrollo cultural de muchos países del mundo.

El pensamiento del presidente Figueres fue muy

\footnotetext{
${ }^{1}$ Coordinador de Entrenamiento e Intercambio Técnico Científico del ICTA, Guatemala.

2 Profesor Asociado e Investigador en Maíz y Trigo de la Universidad de Costa Rica.

${ }^{3}$ Representante de la Compañía Dekalb Pfizer Genetics para Centroamérica y Panamá.

Publicado en Agronomía Mesoamericana, Vol. 1 (1990)
} 
claro al referirse también a la cooperación internacional como medio para la resolución de la problemática regional de nuestra agricultura, pensamiento altamente coincidente con el del grupo de los 61 ya referidos, pues en ese entonces se consideraba la Ayuda Técnica Internacional, como un concepto moderno, pues ya se contaba con otros programas de esta naturaleza como el de las Naciones Unidas, el del Gobierno Federal de Estados Unidos, mediante el llamado Punto Cuarto del Presidente Truman, a través de organismos sumamente interesantes, características también de esa época. También dijo: "Tenemos esfuerzos paralelos, tendientes a difundir en todos nuestros países los conocimientos científicos alcanzados en cualesquiera de ellos". Esa es una nueva concepción moderna de nuestro siglo. Para atrás, lo corriente era ocultado, mantener como secreto los descubrimientos científicos. Hoy solamente en aspectos de guerra se tienen esas precauciones y en toda la vida civil de las naciones la tendencia es a considerar los conocimientos científicos como patrimonio general de la humanidad, a comunicamos los unos con los otros, a evitar el innecesario de repetir las mismas investigaciones $y$, en fin, de procurar el nivelamiento de todos los hombres, el nivel de todos los pueblos, a través de nivelamiento de todas las culturas. En esto estamos aquí reunidos. Por el nivelamiento de las culturas. Estamos para escuchar a los científicos de uno y otro país, de una y otra América. Estamos disfrutando de una ayuda económica de una fundación humanitaria, la Fundación Rockefeller, que como en otras actividades, se propone lo mismo que el Punto Cuarto, lo mismo que la asistencia técnica de las Naciones Unidas: Nivelar la cultura universal.

\section{SEGUNDA REUNIÓN DEL PCCMM COSTA RICA, 1955}

De acuerdo con el programa elaborado por el Comité Organizador de esta segunda reunión, se discutieron las metodologías existentes sobre mejoramiento y de manejo de maíz, a fin deque los asistentes pudieran adoptar la metodología más adecuada a las circunstancias de su país, sin descuidar la metodología y estrategias de la producción de semilla mejorada, ya sea por los propios programas nacionales o por la participación de la iniciativa privada, o entre ambas. Fue necesario fomentar la comunicación con la impresión de glosarios de terminología usada por agricultores de cada país, para evitar problemas de semántica adoptando el Sistema Métrico Decimal como unidad de medida oficial del PCCMCA.

Esta reunión también se caracterizó por el desarrollo de todas las actividades programadas bajo un sentimiento de amplia solidaridad, compañerismo y con un espíritu de alta comprensión humana, en una palabra, de alta camaradería.

\section{TERCERA REUNION DEL PCCMM GUATEMALA, 1956}

Con la celebración de la Tercera Reunión del PCCMM en Guatemala, se abre otro capítulo, consistente en el interés para que en forma rotativa se celebre en un país diferente este cónclave científico.

En esta tercera Reunión se contó con la presencia de personalidades como el Dr. J.C. Harrar, Dr. Lindsley Kimball, así como con la asistencia del Dr. Ralph H. Alle, Director del Instituto de Ciencias Agrícolas de Turrialba, del Dr. Wilson Popenoe, Director de la Escuela Agrícola Panamericana. Se contó asimismo, con la presencia del Dr. Paul Weatherwax de la Universidad de Indiana, quien disertó sobre sus teorías del origen del maíz.

En esta reunión, el Comité Organizador se preocupó porque la misma fuera un éxito, por eso mismo, aunado a ese entusiasmo, la Universidad de San Carlos de Guatemala, le otorgó el honroso título de Profesores Honorarios a los Doctores George Harrar, Edwin de J. WeIlhausen de la Fundación Rockefeller y al Dr. Irwin E. Melhus de Iowa State College, conocido científico maicero. Este acto académico tuvo su escenario en un salón de la Universidad de San Carlos, con la presencia de distinguidos invitados nacionales, y de algunos visitantes, quienes participaron en este justo reconocimiento a estos hombres de ciencia, paladines de la cooperación internacional.

En esta reunión se preparó un buen programa para dar a conocer a los asistentes en forma enfática, una amplia información sobre el estado general de los Programas de Mejoramiento en cada uno de los países participantes y el establecimiento de nuevas estrategias a desarrollar en la próxima reunión.

Hemos escrito algo de lo sucedido en las tres primeras reuniones del PCCMCA que se consideran claves en la creación del PCCMCA, pero, qué paso de la IV a la XXXV reunión? Creemos que lo más conveniente es resumirlo en aquellos aspectos de mayor trascendencia.

\section{OBJETIVOS Y LOGROS OBTENIDOS MÁS IMPORTANTES}

Los objetivos de este programa cooperativo Internacional fueron: Fortalecer a los programas nacionales de producción para alimentar a la población creciente de cada país, que para el caso de Centroamérica significa producir para el año 2,000 las necesidades energéticas y proteínicas de unos 40 millones de habitantes. Para realizar esto es necesario aumentar la producción del maíz mediante el uso de semillas y prácticas culturales mejoradas; producir e intercambiar información experimental y de índole relacionada con el cultivo del maíz; levantar el nivel técnico del personal trabajando con el mejoramiento del maíz en Centroamérica. Estos objetivos han 
sido alcanzados en mayor o menor medida en el transcurso de los 36 años de trabajo del PCCMCA, como lo atestiguan: La amplia y documentada publicación y difusión de los trabajos experimentales de maíz, frijol, arroz, sorgo y otros cultivos, cerca de 4,000 documentos están registrados en los informes de actividades del PCCMCA en 36 años de trabajo ininterrumpido. El incremento de ${ }_{i}$ nivel técnico de los encargados de la labor experimental, de difusión y producción de semillas de granos básicos y más de 100 técnicos recibieron entrenamiento y cursos avanzados en educación agrícola. Finalmente, el aumento en la productividad en cada uno de los cultivos básicos, se debe en medida importante a no dudarlo a las labores del PCCMCA. Los resultados conseguidos con el trabajo del PCCMCA se deben en parte a los Ministerios de Agricultura y Universidades de Centroamérica y a las instituciones internacionales dedicadas al progreso de la agricultura en el área, tales como: La Fundación Rockefeller, CIMMYT, IICA, CIAT, ICRISAT, IRRI, FAO, CATIE, AID, INCAP y otras que desarrollan funciones en Centroamérica.

El apoyo material y moral de los mencionados organismos fue de primera importancia en dar el estímulo inicial y el apoyo consistente a través de los años. Pero no lo fue menos la participación con espíritu cooperativo, voluntario y desinteresado, de los técnicos agrícolas centroamericanos y de países vecinos como México, Colombia y Venezuela, que en última instancia fueron el alma del PCCMCA.

Cabe destacar que desde 1954 hasta 1989, se han realizado 35 reuniones anuales del PCCMCA, en forma rotativa en todos las países de Centroamérica y El Caribe. En Costa Rica se celebraron 8, en Honduras 6, en Panamá 5, en Nicaragua 4, en El Salvador 6, en Guatemala 5 y en República Dominicana 1.

De una asistencia de 61 delegados a la primera reunión realizada en Costa Rica durante el año 1954, pasó a 500 delegados en la reunión efectuada en Honduras en 1989.

Durante los años de 1954 a 1961 se inició la prueba de posiblemente casi todo el material genético de maíz existente en Centroamérica y El Caribe. Con base en esta intensa labor de prueba de variedades de maíz, fue posible introducir en cada país de Centroamérica una o más variedades mejoradas o híbridos, de los que se produjo semilla comercial en diferente medida para el uso de los agricultores.

En 1960, el PCCMCA inició el mejoramiento genético de maíz usando la técnica de Selección Masal Modificada, habiéndose conseguido desarrollar un número de variedades mejoradas de amplia difusión en cada país. La Fundación Rockefeller inició también en este período un programa de entrenamiento y de estudios avanzados para los técnicos que trabajaban en maíz en Centroamérica. Como resultado de esta labor fue posible contar con personal de mayor nivel técnico para los trabajos de mejoramiento, de producción de semillas y para otras actividades relacionadas con la producción de maíz.

Las personas que jugaron papel protagonista en este período fueron: Dr. Edwin de J. WeIlhausen, Dr. Sterling Wortman y Dr. Robert D. Osler de la Fundación Rockefeller, destacados en la Oficina de Estudios Especiales de la Secretaría de Agricultura de México. El primer Coordinador del PCCMCA fue el Dr. Alfredo Carballo Q., técnico costarricense que fungió como tal entre 1954 hasta 1959. A él le correspondió la labor de dar forma al PCCMCA. A partir de 1960 se hizo cargo de la coordinación del programa el Ing. Angel Salazar B., quien trabajó hasta el año de 1967. Su labor consistió en consolidar el PCCMCA y en expandir su área de acción de sólo maíz a los cuatro granos básicos de Centroamérica, como lo son: Maíz, Arroz, Sorgo y Frijol.

En 1962, el cultivo del frijol fue incluido en el PCCMCA. Desde este año hasta la fecha, la labor con este cultivo siguió en forma paralela a la del maíz, es decir, dedicó sus esfuerzos al mejoramiento genético de variedades, desarrollo de prácticas mejoradas de cultivo y en general, al estudio y documentación de los problemas que atañen a este cultivo, tan importante en la dicta de la población de Centroamérica.

El cultivo de arroz fue introducido al PCCMCA en 1965 y el cultivo del sorgo se adhirió finalmente en 1966, con lo que los 4 granos básicos de la alimentación humana de Centroamérica quedaron bajo la atención del PCCMCA.

En el período de 1962 a 1966, la labor de prueba de variedades de maíz continuó mediante los ensayos uniformes de rendimiento que incluían todos los maíces mejorados del área y de los países tropicales vecinos. También los trabajos de mejoramiento genético continuaron con el uso de la Selección Recurrente en sus diferentes variantes. Asimismo, se realizaron trabajos en mejoramiento de las prácticas culturales del maíz, tales como: Control de malezas; insectos y enfermedades, así como prácticas de fertilización y densidades de población y el uso de diversos métodos de siembra en maíz y otros cultivos.

En 1968, se hizo cargo de la coordinación del PCCMCA el Dr. Willy Villena, miembro del personal del CIMMYT, con sede en México.

En el período comprendido entre 1968 a 1976, el PCCMCA concentró sus esfuerzos en el mejoramiento de los cuatro granos básicos; en la elevación del nivel técnico del personal trabajando con estos cultivos, mediante becas de entrenamiento y de estudios avanzados, en el estímulo de la producción de semillas mejoradas en cantidades comerciales en cada país del istmo centroamericano. Las empresas privadas de semillas también ingresaron a este mercado durante este período de tiempo.

En el año 1977 asistieron por primera vez delegados de los programas de mejoramiento de Hortalizas, 
Raíces y Tubérculos de Centroamérica. Al año siguiente, 1978 , asistieron también delegados de los programas de frutales. Esto significó una nueva expansión del área de influencia del PCCMCA, así como el aumento del volumen de los trabajos presentados en las reuniones anuales. Nada impidió, sin embargo, que cada año se realizaran con éxito las reuniones del PCCMCA. Los problemas políticos, económicos, telúricos (terremotos), atmosféricos (huracanes), no fueron causa de interrupción de las reuniones, ni de los trabajos del PCCMCA. En este período también varias instituciones internacionales de cooperación agrícola ofrecieron sus buenos oficios para institucionalizar el PCCMCA, o por lo menos, para formalizarlo. Tampoco estos esfuerzos, bien intencionados, redujeron la actividad ni el entusiasmo de los participantes en el PCCMCA. Así, este singular programa siguió su trayectoria de manera informal, voluntaria y cooperativa.

Entre 1979 y 1981, se agregaron al PCCMCA los programas de Sistemas de Producción de Granos Básicos y Producción Animal, así como los programas de Producción de Semilla. Así, no sólo se abarcó el área agronómica de los cultivos más importantes del área, sino también el de la Producción Animal. Para finales de la década de los 70, dentro del PCCMCA, ya se contaba con personal calificado para la tarea de mejoramiento, comunicación, técnicas de producción, semillas y otras áreas relacionadas, con lo que la labor de los participantes del programa fue más eficiente y productiva. Todos los países participantes en el PCCMCA contaban ya en 1980, con programas bien establecidos de mejoramiento genético de los granos básicos, así como de servicios de estadística, comunicación, certificación de semilla, producción de semilla y otros. Prácticamente, cada país de Centroamérica contaba ya con variedades mejoradas e híbridos de maíz y sorgo, y con variedades mejoradas de frijol y arroz, ya sea localmente desarrolladas o introducidas de otros países del área, o de países vecinos a Centroamérica.

En los años 1982 a 1984, nuevo personal altamente calificado se agrega a los programas locales de mejoramiento de los granos básicos de Centroamérica. Con ello, la labor de investigación y presentación de trabajos se incrementó en volumen y calidad. La selección de las presentaciones magistrales, como la de los ponentes se hacen considerando que sean de interés general y de actualidad en actividades de la ciencia agropecuaria de la región. Se establecen tres reconocimientos para los tres mejores trabajos por cada mesa, conside- rando su calidad científica y presentación. Estos reconocimientos se dan extendiendo plaquetas (platones) y/o diplomas (pergaminos).

Fue también en 1982 que el CATIE, a través de su departamento de Producción Vegetal, y patrocinado por el FIDA, publica la Bibliografía del PCCMCA desde 1954 hasta 1982

En 1985 el Dr. Willy Villena deja la Coordinación del PCCMCA al Ing. Hugo Córdova y con él se inicia el actual período del PCCMCA. En esta etapa se agregan aún otros programas más al PCCMCA y son el de Recursos Fitogenéticos y el de Oleaginosas. En el área de mejoramiento del maíz se inició la formación de híbridos con alta producción y resistencia a enfermedades, esto con base en líneas de líneas desarrolladas en forma cooperativa por el PCCMCA y el ICTA o en los programas locales de mejoramiento. La actividad de producción de semilla y la certificación con técnicos calificados se estableció en el área. En lo que a reconocimientos de presentación de trabajos se refiere, se amplía en el sentido de otorgar a los dos mejores trabajos del PCCMCA del año anterior, un premio de \$U.S. 150 cada uno y un premio de $\$$ U.S. 500 al científico más distinguido. Por otra parte, se implanta una nueva modalidad y es la exhibición de Poster de los trabajos de mayor importancia que llegan de cada uno de los países.

Al final de la década de los años 80 puede decirse que el PCCMCA es un programa maduro, sólido y que tiene en su haber, resultados cuyo beneficio es evidente. En efecto, debe ser difícil encontrar un agricultor que no use actualmente uno o varios de los resultados de la labor de investigación en maíz, frijol, arroz o sorgo. Varios técnicos que participaron de las actividades del PCCMCA, o que recibieron entrenamiento o educación superior ocupan u ocuparon cargos directivos en la investigación y administración agrícola de Centroamérica. La documentación presentada en las 35 reuniones anuales está publicada y constituye bibliografía de uso inevitable en toda actividad relacionada con los cultivos básicos de Centroamérica y El Caribe.

$\mathrm{Si}$ alguien cree que no es posible organizar un programa cooperativo, sin que halla convenios institucionales formales, fondos especiales presupuestados, y que sólo cuenta con la voluntad de sus cooperadores y el apoyo de instituciones locales e internacionales que dejan hacer lo que es posible en el medio, ahí está como ejemplo el Programa Cooperativo Centroamericano y del Caribe para el Mejoramiento de Cultivos Alimenticios y Producción Animal. 